\title{
Computed Tomography Pulmonary Angiography during Pregnancy: Radiation Dose of Commonly Used Protocols and the Effect of Scan Length Optimization
}

\author{
Babs M.F. Hendriks, MD ${ }^{1,2}$, Roald S. Schnerr, $\mathrm{PhD}^{1}$, Gianluca Milanese, MD ${ }^{1,3}$, \\ Cécile R.L.P.N. Jeukens, $\mathrm{PhD}^{1}$, Sandra Niesen, $\mathrm{RT}^{1}$, Nienke G. Eijsvoogel, $\mathrm{MD}^{1,2}$, \\ Joachim E. Wildberger, MD, PhD ${ }^{1,2}$, Marco Das, MD, PhD ${ }^{1,4}$
}

\begin{abstract}
${ }^{1}$ Department of Radiology \& Nuclear Medicine, Maastricht University Medical Centre, Maastricht, The Netherlands; ${ }^{2}$ CARIM School for Cardiovascular Diseases, Maastricht University Medical Centre, Maastricht, The Netherlands; ${ }^{3}$ Department of Surgical Sciences, Division of Radiology, University of Parma, Parma, Italy; ${ }^{4}$ Department of Diagnostic and Interventional Radiology, Duisburg, Germany
\end{abstract}

Objective: To evaluate the radiation dose for pregnant women and fetuses undergoing commonly used computed tomography of the pulmonary arteries (CTPA) scan protocols and subsequently evaluate the simulated effect of an optimized scan length. Materials and Methods: A total of 120 CTPA datasets were acquired using four distinctive scan protocols, with 30 patients per protocol. These datasets were mapped to Cristy phantoms in order to simulate pregnancy and to assess the effect of an effective radiation dose (in $\mathrm{mSv}$ ) in the first, second, or third trimester of pregnancy, including a simulation of fetal dose in second and third trimesters. The investigated scan protocols involved a 64-slice helical scan at $120 \mathrm{kVp}$, a high-pitch dual source acquisition at $100 \mathrm{kVp}$, a dual-energy acquisition at 80/140 kVp, and an automated-kV-selection, high pitch helical scan at a reference $\mathrm{kV}$ of $100 \mathrm{kV}_{\text {ref. }}$. The effective dose for women and fetuses was simulated before and after scan length adaptation. The original images were interpreted before and after scan length adaptations to evaluate potentially missed diagnoses. Results: Large inter-scanner and inter-protocol variations were found; application of the latest technology decreased the dose for non-pregnant women by $69 \%(7.0-2.2 \mathrm{mSv})$. Individual scan length optimization proved safe and effective, decreasing the fetal dose by $76-83 \%$. Nineteen $(16 \%)$ cases of pulmonary embolism were diagnosed and, after scan length optimization, none were missed.

Conclusion: Careful CTPA scan protocol selection and additional optimization of scan length may result in significant radiation dose reduction for a pregnant patient and her fetus, whilst maintaining diagnostic confidence.

Keywords: Computed tomography; Pulmonary angiography; Radiation dose; Pregnancy; Fetus; Pulmonary embolism

\section{INTRODUCTION}

Pulmonary embolism (PE) is a rare but dangerous complication of pregnancy and the post-partum period (1-3). PE is a manifestation of venous thromboembolic
(VTE) disease and typically originates from deep venous thrombosis. Since normal pregnancy is accompanied by a state of hypercoagulability and hypofibrinolysis, the overall risk of developing VTE is five-fold greater than that in nonpregnant women, with the highest risk occurring in the

Received December 29, 2017; accepted after revision July 24, 2018.

BMFH, RSS, GM, NGE, CRPLNJ and SN have nothing to disclose.

JEW reports institutional research grants from Siemens, Philips, Bayer, AGFA and personal fees from Siemens, Bayer, outside the submitted work.

MD reports institutional research grants (MUMC) from Siemens, Philips, Bayer, and personal fees from Siemens, Bayer, outside the submitted work.

Corresponding author: Babs M.F. Hendriks, MD, Department of Radiology \& Nuclear Medicine, Maastricht University Medical Centre, P. Debyelaan 25 P0 Box 5800, 6202 AZ Maastricht, The Netherlands.

- Tel: (3143) 3874911 Fax: (3143) 3877093•E-mail: babs.hendriks@mumc.nl

This is an Open Access article distributed under the terms of the Creative Commons Attribution Non-Commercial License (https:// creativecommons.org/licenses/by-nc/4.0) which permits unrestricted non-commercial use, distribution, and reproduction in any medium, provided the original work is properly cited. 
post-partum period $(4,5)$. Unfortunately, the non-specific clinical symptoms of PE such as chest pain and dyspnea mimic certain symptoms of pregnancy, often leaving clinicians unsure of whether to pursue diagnostics.

The American Thoracic Society (ATS) recommends performing bilateral venous compression ultrasound of the lower extremities, followed by further testing if negative. The placement of computed tomography of the pulmonary arteries (CTPA) in the diagnostic algorithm for pregnant patients is currently firmly established; the ATS recommends ventilation/perfusion (V/Q) scanning in case of a normal chest radiography and CTPA in case of an abnormal chest radiography (6). The Australasian Society of Thrombosis and Heamostasis, together with the Councils of the Society of Obstetric Medicine of Australia and New Zealand recommend CTPA when V/Q scanning is not available or non-diagnostic (2). However, all current recommendations are based on limited scientific evidence and lack clear recommendations with regard to the scan protocol $(2,6,7)$. This indicates a need for further research regarding PE imaging diagnostics in pregnant women, which is underlined by the variety of CTPA scan protocols and corresponding radiation doses found in the literature for this patient group $(6,8-10)$.

The current trend of individualized diagnostics has led to increasingly patient-tailored CTPA scan protocols, thereby lowering both contrast material (CM) doses and radiation doses to an all-time low (11-17). Pregnant patients might benefit most from radiation dose reduction due to the rapid proliferation of tissues in both the fetus and expectant mother $(18,19)$.

This study aimed to quantify the differences in radiation dose when applying various CTPA scan protocols on three currently, clinically used CT scanners. Two methods for adapting the scan length were then investigated; a 10\% reduction in the caudal scan range and an individually optimized scan range along the $z$-axis and radiation dose were evaluated. Finally, the technician's decision was evaluated before and after observing the results of this study.

\section{MATERIALS AND METHODS}

\section{Study Population and Simulation}

Thirty consecutive female patients undergoing CTPA were included for each of the four scan protocols, resulting in a total of 120 patients being included in this study. The mean patient ages for the four consecutive protocols were $49 \pm$ $20,63 \pm 15,57 \pm 16$, and $55 \pm 18$ years. All patients had a clinical suspicion of PE based on their history and clinical symptoms. A waiver of written informed consent was obtained from the local ethical committee (approval of the local Medical Ethical Review Board [METC] was obtained; waiver reference numer 14-4-158.4). The real patient data were used as the basis for computer-guided phantom simulations, thus assuring that the phantoms would carry the natural variance in body habitus of a real CTPA patient group.

All 120 patients were non-pregnant women; after initial organ dose estimations were performed, the three stages of pregnancy were simulated. This process (described in the next paragraph) resulted in an additional 360 phantom simulations, i.e., $\mathrm{n}=120$ per pregnancy trimester. For the 2 nd and 3rd trimester of pregnancy, fetuses were also simulated in the software; this resulted in 240 phantom fetuses ( $n=120$ per trimester) in total, for whom the dose estimations are provided, see Figures 1 and 2.

Using a dose monitoring software (Radimetrics ${ }^{\mathrm{TM}}$ Enterprise Platform, Bayer Healthcare, Berlin, Germany), the original 120 patients were mapped to mathematical anthropomorphic (Cristy) phantoms based on age, sex, and size, which was determined from the CT scan and scout images (20). This software allows for modification of the patient characteristics and scan parameters such as scan range, sex, and pregnancy status to simulate their effect

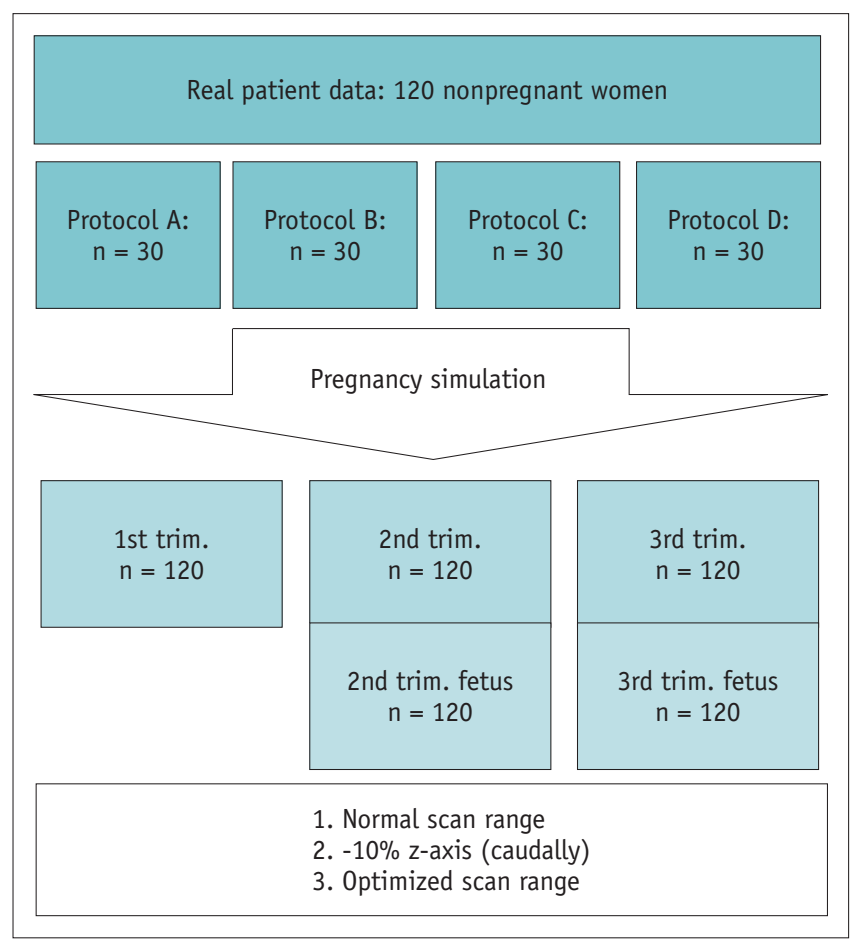

Fig. 1. Phantom simulation flow chart explaining simulation process. trim. $=$ trimester 
on organ (equivalent) dose, effective dose, and fetal dose (Fig. 2). The Monte Carlo code employed by the software simulates the $\mathrm{X}$-ray source and radiation transport inside the human body, including scattered radiation (21). The dose monitoring software takes dose modulation along the z-axis into account; however, it does not register angular dose modulation, thereby assuming equal distribution of dose over the slice. None of the scan protocols investigated used angular dose modulation.

\section{Scan and Injection Protocol}

The three CT scanners investigated in this study were a 64-slice multidetector-row CT (MDCT; Brilliance 64, Philips Healthcare, Best, The Netherlands), a 2nd generation dual-
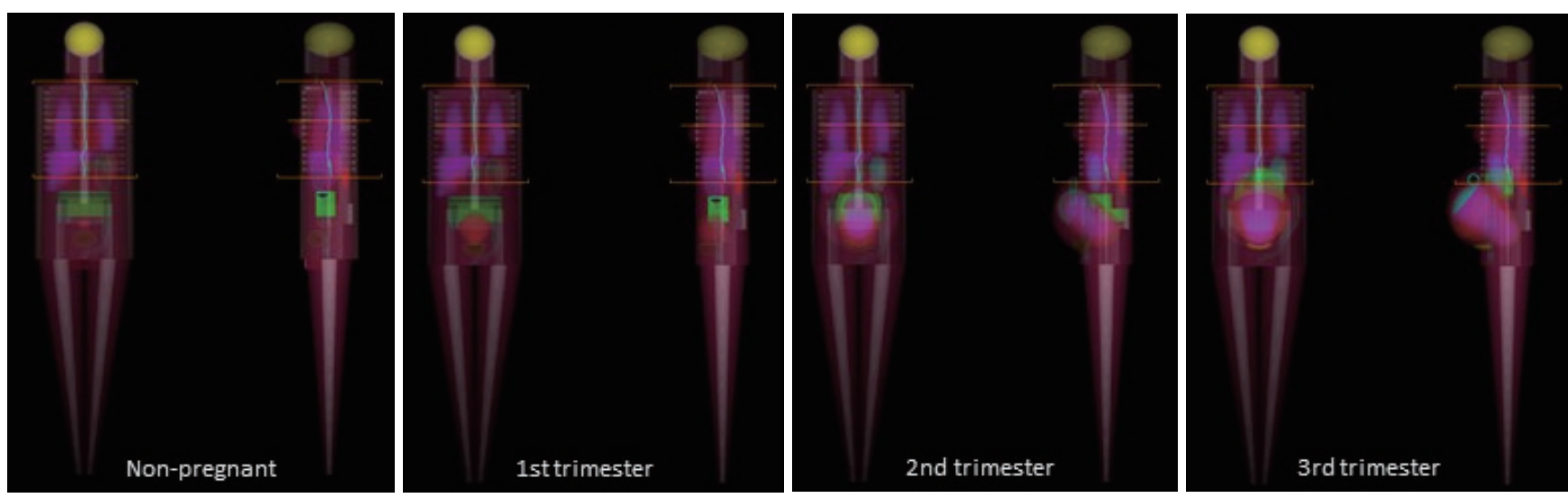

Fig. 2. Software phantom simulations. Phantoms in this Figure are modeled on 37-year-old woman, first presented as non-pregnant female (left) and subsequently simulated in all three pregnancy stages. Red lines indicate original scan length.

Table 1. Scan and Injection Protocol Overview

\begin{tabular}{|c|c|c|c|c|}
\hline Scan Protocol & $A$ & B & C & D \\
\hline \multicolumn{5}{|l|}{ Scan technique } \\
\hline Scanner & 64-slice MDCT & $2 \times 128$-slice DSCT & $2 \times 128$-slice DSCT & $2 \times 192$-slice DSCT \\
\hline \multirow[t]{2}{*}{ Automated tube voltage selection } & Off & Off & Off & $0 n$ \\
\hline & & & Tube B & \\
\hline Tube voltage & $120 \mathrm{kV}$ & $100 \mathrm{kV}$ & $140 \mathrm{kV}$ & $100 \mathrm{kV}_{\text {ref }}$ \\
\hline kV-range & & & & $70-120$ \\
\hline Dose modulation & & & ON & \\
\hline Tube current & $200 \mathrm{mAs}_{\text {ref }}$ & $250 \mathrm{mAs}_{\mathrm{ref}}$ & $275 \mathrm{mAs}_{\text {ref }}$ & $105 \mathrm{mAs}_{\text {ref }}$ \\
\hline Pitch & 0.9 & 2.6 & 0.9 & 1.2 \\
\hline Collimation & $0.625 \mathrm{~mm}$ & $0.6 \mathrm{~mm}$ & $0.6 \mathrm{~mm}$ & $0.6 \mathrm{~mm}$ \\
\hline \multicolumn{5}{|l|}{ CM injection } \\
\hline Concentration & \multicolumn{4}{|c|}{$300 \mathrm{mgI} / \mathrm{mL}$} \\
\hline Timing & \multicolumn{4}{|c|}{ Test bolus } \\
\hline Main bolus volume & $75 \mathrm{~mL}$ & $44-69 \mathrm{~mL}^{*}$ & $42-78 \mathrm{~mL}^{*}$ & $21-61 \mathrm{~mL}^{\dagger}$ \\
\hline Flow rate & $6.0 \mathrm{~mL} / \mathrm{s}$ & $5.0-8.6 \mathrm{~mL} / \mathrm{s}^{*}$ & $4.2-7.8 \mathrm{~mL} / \mathrm{s}^{*}$ & $2.6-7.6 \mathrm{~mL} / \mathrm{s}^{\dagger}$ \\
\hline Total iodine load & $22.5 \mathrm{gI}$ & $19.2-26.7 \mathrm{gI}^{*}$ & $18.6-29.4 \mathrm{gI}^{*}$ & $8.4-24 \mathrm{gI}^{\dagger}$ \\
\hline \multicolumn{5}{|l|}{ Reconstruction } \\
\hline $\mathrm{FBP} / \mathrm{IR}^{\dagger}$ & FBP & FBP & FBP & ADMIRE 3 \\
\hline Slice thickness & \multicolumn{4}{|c|}{$1.0 \mathrm{~mm}$} \\
\hline Increment & $0.7 \mathrm{~mm}$ & $0.8 \mathrm{~mm}$ & $0.8 \mathrm{~mm}$ & $0.7 \mathrm{~mm}$ \\
\hline Kernel & B & B30f & B30f/D30f & B40v \\
\hline
\end{tabular}

* Range of values is given; injection protocol was adapted for patient body weight as determined by contrast injection software (Certegra ${ }^{\mathrm{TM}}$ P3T, Bayer Healthcare), with standard injection time of 8 seconds for protocol B and 10 seconds for protocol C, ${ }^{\dagger}$ Patient body weight tailored protocol was subsequently adapted to account for kV-related attenuation changes, by adding or subtracting $10 \%$ iodine-delivery rate per $10 \mathrm{kV}$ step up or down. Injection time was standardised at 8 seconds. Detailed description of this kV-based injection protocol was recently published (41). CM = contrast material, DSCT $=$ dual-source $\mathrm{CT}, \mathrm{FBP}=$ filtered back projection, $\mathrm{IR}=$ iterative reconstruction, $\mathrm{MDCT}$ = multidetector-row $\mathrm{CT}$ 
source CT (DSCT; SOMATOM, Definition Flash, Siemens Healthineers, Forchheim, Germany), and a 3rd generation DSCT (SOMATOM Force, Siemens Healthineers).

The following four scan protocols were investigated: standard helical acquisition on the 64-slice MDCT (referred to in this text as ' $A$ '), both a high pitch 'flash' helical scan and a dual-energy acquisition (respectively referred to in this text as ' $\mathrm{B}$ ' and ' $\mathrm{C}$ ') on the 2nd generation DSCT, and a high pitch helical scan using patient- and indicationtailored automated tube voltage selection (ATVS; CARE kV, Siemens Healthineers) on the 3rd generation DSCT (referred to in this text as ' $D$ ').

An overview of all scan parameters of the four protocols is listed in Table 1. All patients were scanned using the breath-hold technique and arm abduction. Careful instructions were given to avoid the Valsalva effect (22). The cranio-caudal scan range was set by the technicians, covering the entire thorax and extending to include the costodiaphragmatic recess, which was identical to a standard thoracic $\mathrm{CT}$ scan range.

In all cases, pre-warmed $\left(37^{\circ} \mathrm{C} / 99^{\circ} \mathrm{F}\right) \mathrm{CM}$ was used; iopromide $300 \mathrm{mgI} / \mathrm{mL}$ (Bayer Healthcare). In order to determine scan delay, the test bolus technique was applied with a $30 \mathrm{~mL}$ saline flush at the same flow rate as the CM. The CM was injected into the left or right antecubital vein using 18-20 G needles and flushed with $40 \mathrm{~mL}$ of saline after the main CM bolus (Table 1 ).

\section{Adaptation of Scan Range}

Two methods of reducing z-axis coverage were simulated and investigated. First, the original scan range was reduced by a fixed $10 \%$ at the caudal end of the scan. The second method involved optimizing the scan range on a per-patient basis. This was achieved by setting the new scan range from the lung apex to the top of the most caudal diaphragm on the scout image (Fig. 3).

\section{Effective Dose Assessment}

Dose monitoring software was used to determine the effective doses in mSv per scan, calculated according to the International Commission on Radiological Protection, Publication 103 (ICRP 103) (23). Organ-specific (equivalent) doses were assessed for the thymus, thyroid, lungs, and breast tissue in all 120 non-pregnant female simulations. The effective dose and breast tissue equivalent dose were assessed for all stages of pregnancy, and the fetal dose was calculated for the second and third trimester of pregnancy.

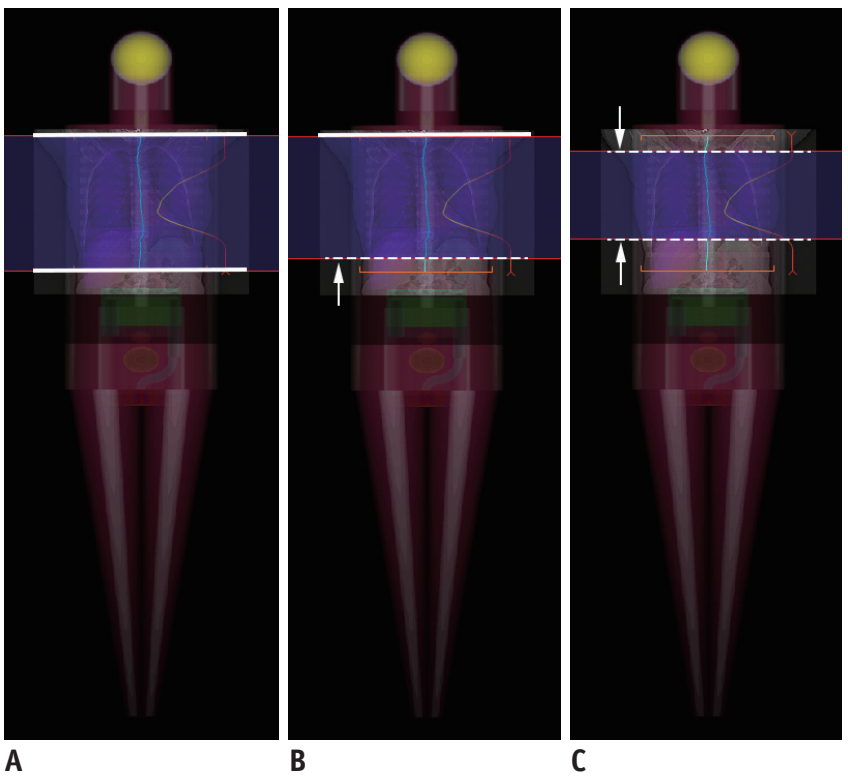

Fig. 3. Scan length adaptation. 66-year-old woman who underwent computed tomography of pulmonary arteries was mapped to nonpregnant female Cristy phantom according to body habitus and age. A. Two straight white lines show original scan range, and dose modulation is projected vertically over image. B. Dotted white line (arrow) indicates simulated scan range after 10\% caudal range reduction. C. Dotted white lines indicate individual scan range (arrows) optimization; lung apex to top of most caudal diaphragm.

All assessments were then repeated for both scan range optimization techniques.

\section{Diagnostic Assessment and Image Quality}

All 120 original scans were evaluated on a dedicated workstation. An experienced researcher delineated regions of interest in the pulmonary trunk and both pulmonary arteries in order to evaluate the intravascular enhancement. Mean enhancement levels were calculated and values of $>180 \mathrm{HU}$ were considered diagnostic (24). The presence, location, and extent of PE was reported by thoracic radiologists with 4-25 years' experience of reporting CTPA scans. Any incidental findings or alternative diagnoses were reported both before and after adaptation along the z-axis; the latter was performed by noting the top and bottom slice number after adaptation and only reading the slices in between.

\section{Assessment of Clinical Practice}

As a second step, eight qualified CT technicians (1-3 years' experience) were asked to set the scan range on 12 scout views of previously scanned pregnant patients as though they were about to perform a CTPA. The technicians were informed of the clinical question - suspected PE - 
and of the pregnancy status, including the trimester. The scan ranges were noted as the most cranial and most caudal slice. After completion of the dose simulation study, the same technicians were informed of the results of our simulation and asked to repeat the exercise.

\section{Statistical Analysis}

Data analysis was conducted using the statistical package for the social sciences (SPSS) version 23.0 (IBM Corp., Armonk, NY, USA). For continuous variables, group means were compared using a one-way analysis of variance (ANOVA) and a post hoc Tukey's test was applied to analyze differences between groups in case of a significant result in the one-way ANOVA. All $p$ values were two-sided and a $p$ value of $<0.05$ was considered statistically significant. Continuous values are reported as mean \pm standard deviation.

\section{RESULTS}

\section{Original Study Population}

The patient groups consisted of 30 consecutive nonpregnant women in each protocol. The mean patient age was significantly lower in patients in group $A$ than in those in group $\mathrm{B}(p=0.010)$.

For six patients, there were no data available on body weight ( $n=4$ in group $A$ and $n=2$ in group $C$ ). The mean body weight per group was as follows: $81.4 \pm 23.9 \mathrm{~kg}$ for protocol A, $71.2 \pm 13.0 \mathrm{~kg}$ for protocol $\mathrm{B}, 68.8 \pm 19.9 \mathrm{~kg}$ for protocol C, and $73.7 \pm 20.4 \mathrm{~kg}$ for protocol $D$. The mean body weight did not differ significantly between protocol groups $(p=0.104)$.
Protocol D employed ATVS, which primarily resulted in a $\mathrm{kV}$ selection between $70 \mathrm{kV}$ and $90 \mathrm{kV}(\mathrm{n}=2$ for $120 \mathrm{kV}, \mathrm{n}=$ 6 for $90 \mathrm{kV}, \mathrm{n}=19$ for $80 \mathrm{kV}$, and $\mathrm{n}=3$ for $70 \mathrm{kV}$ ).

\section{Simulated Dose Assessment and Adaptation of Scan Range}

The mean effective doses per protocol for the nonpregnant women using the standard scan range were as follows: $7.0 \pm 1.5 \mathrm{mSv}$ for $\mathrm{A}, 4.4 \pm 1.0 \mathrm{mSv}$ for $\mathrm{B}, 7.8 \pm 0.8$ $\mathrm{mSv}$ for $\mathrm{C}$, and $2.2 \pm 1.2 \mathrm{mSv}$ for $\mathrm{D}(p<0.01)$ (Table 2). The thymus, lungs, and breasts all received similar organ doses before and after scan range adaptation, since these remained within the scan range. The doses were highest for protocol $\mathrm{C}$ and lowest for protocol $\mathrm{D}$ (Table 3).

Effective patient dose increased slightly throughout the pregnancy trimesters for each protocol; the dose increased from the 1 st to the 3 rd trimester from $2.2 \%$ to $5.5 \%$. This effect was also observed for the fetus, but the increase was more significant; the dose increase from the 2 nd to the $3 \mathrm{rd}$ trimester was $37 \%, 41 \%, 40 \%$, and $42 \%$ for protocols $A, B, C$, and $D$, respectively (Table 4 ).

The average scan length before adaptation was comparable for most scan protocols; $303 \pm 26 \mathrm{~mm}$ for protocol A, 275 $\pm 36 \mathrm{~mm}$ for $\mathrm{B}, 291 \pm 27 \mathrm{~mm}$ for $\mathrm{C}$, and $293 \pm 32 \mathrm{~mm}$ for $\mathrm{D}$, with a significant baseline scan length difference between protocols A and $\mathrm{B}(p=0.004)$. The individually optimized $z$-axis resulted in a $33 \%, 30 \%, 30 \%$, and $31 \%$ reduction in the scan ranges for protocols $A, B, C$, and $D$, respectively. There was no significant difference in the range reduction between the four protocols $(p>0.438)$ (Fig. 4).

The individually-optimized scan range was successful in terms of radiation dose reduction (Table 2, Fig. 5). For non-

Table 2. Maternal Effective Doses Per Scan Protocol, including Effective Doses after Z-Axis Adaptation

\begin{tabular}{|c|c|c|c|c|c|c|}
\hline Radiation Dose & Pregnancy Stage & Scan Range & Prot. $A(m S v \pm S D)$ & Prot. B $(\mathrm{mSv} \pm \mathrm{SD})$ & Prot. $\mathrm{C}(\mathrm{mSv} \pm \mathrm{SD})$ & Prot. $\mathrm{D}(\mathrm{mSv} \pm \mathrm{SD})$ \\
\hline \multirow{12}{*}{$\begin{array}{l}\text { Maternal dose } \\
\text { (ICRP 103) }\end{array}$} & & Standard & $7.0 \pm 1.5$ & $4.4 \pm 1.0$ & $7.8 \pm 0.8$ & $2.2 \pm 1.2$ \\
\hline & Not pregnant & $-10 \%$ & $6.5 \pm 1.4$ & $4.0 \pm 0.9$ & $7.1 \pm 0.7$ & $2.0 \pm 1.1$ \\
\hline & & Optimal & $5.2 \pm 1.1$ & $3.3 \pm 0.6$ & $5.8 \pm 0.7$ & $1.7 \pm 0.8$ \\
\hline & & Standard & $9.2 \pm 2.8$ & $5.1 \pm 1.3$ & $9.1 \pm 0.8$ & $3.0 \pm 2.2$ \\
\hline & First trimester & $-10 \%$ & $8.5 \pm 2.6$ & $4.6 \pm 1.2$ & $8.2 \pm 0.8$ & $2.7 \pm 2.0$ \\
\hline & & Optimal & $6.7 \pm 2.1$ & $3.8 \pm 0.9$ & $6.7 \pm 0.7$ & $2.2 \pm 1.6$ \\
\hline & & Standard & $9.4 \pm 2.9$ & $5.2 \pm 1.4$ & $9.4 \pm 0.9$ & $3.1 \pm 2.3$ \\
\hline & Second trimester & $-10 \%$ & $8.7 \pm 3.0$ & $4.8 \pm 1.2$ & $8.5 \pm 0.8$ & $2.8 \pm 2.1$ \\
\hline & & Optimal & $6.9 \pm 2.1$ & $3.9 \pm 0.9$ & $6.9 \pm 0.7$ & $2.3 \pm 1.6$ \\
\hline & & Standard & $9.4 \pm 3.0$ & $5.3 \pm 1.4$ & $9.6 \pm 0.9$ & $3.1 \pm 2.3$ \\
\hline & Third trimester & $-10 \%$ & $8.9 \pm 2.8$ & $4.9 \pm 1.3$ & $8.7 \pm 0.8$ & $2.9 \pm 2.1$ \\
\hline & & Optimal & $7.0 \pm 2.2$ & $4.0 \pm 0.9$ & $7.1 \pm 0.8$ & $2.3 \pm 1.7$ \\
\hline
\end{tabular}

ICRP 103 = International Commission on Radiological Protection, Publication 103, SD = standard deviation 
Table 3. Organ Specific (Equivalent) Doses for Non-Pregnant Patients

\begin{tabular}{|c|c|c|c|c|c|}
\hline Organ & Scan Range & Prot. A $(m S v \pm S D)$ & Prot. B $(\mathrm{mSv} \pm \mathrm{SD})$ & Prot. C (mSv \pm SD) & Prot. D $(\mathrm{mSv} \pm \mathrm{SD})$ \\
\hline \multirow{3}{*}{ Thyroid } & Standard & $4.7 \pm 2.6$ & $1.6 \pm 1.6$ & $2.6 \pm 1.0$ & $1.0 \pm 2.1$ \\
\hline & $-10 \%$ & $4.7 \pm 2.6$ & $1.6 \pm 1.6$ & $2.6 \pm 1.0$ & $1.0 \pm 2.1$ \\
\hline & Optimal & $2.6 \pm 1.3$ & $1.2 \pm 1.0$ & $1.8 \pm 0.7$ & $0.4 \pm 0.3$ \\
\hline \multirow{3}{*}{ Thymus } & Standard & $12.8 \pm 2.7$ & $8.7 \pm 1.9$ & $15.7 \pm 1.5$ & $4.4 \pm 1.8$ \\
\hline & $-10 \%$ & $12.7 \pm 2.6$ & $8.7 \pm 1.9$ & $15.6 \pm 1.5$ & $4.4 \pm 1.8$ \\
\hline & Optimal & $12.5 \pm 2.6$ & $8.5 \pm 1.8$ & $14.9 \pm 2.1$ & $4.2 \pm 1.7$ \\
\hline \multirow{3}{*}{ Lungs } & Standard & $12.9 \pm 2.7$ & $8.6 \pm 1.9$ & $15.7 \pm 1.4$ & $4.2 \pm 1.9$ \\
\hline & $-10 \%$ & $12.8 \pm 2.7$ & $8.5 \pm 1.8$ & $15.4 \pm 1.3$ & $4.2 \pm 1.9$ \\
\hline & Optimal & $12.1 \pm 2.5$ & $8.1 \pm 1.7$ & $14.4 \pm 1.7$ & $3.9 \pm 1.7$ \\
\hline \multirow{3}{*}{ Breasts } & Standard & $13.3 \pm 3.5$ & $8.5 \pm 2.1$ & $15.1 \pm 0.8$ & $4.8 \pm 2.6$ \\
\hline & $-10 \%$ & $13.2 \pm 3.5$ & $8.5 \pm 2.0$ & $15.0 \pm 0.8$ & $4.8 \pm 2.6$ \\
\hline & Optimal & $13.0 \pm 3.5$ & $8.4 \pm 2.0$ & $14.8 \pm 0.7$ & $4.7 \pm 2.5$ \\
\hline
\end{tabular}

Table 4. Fetal Dose before and after Z-Axis Adaptation, Per Scan Protocol

\begin{tabular}{lcccccc}
\hline Radiation Dose & Pregnancy Trimester & Scan Range & Prot. A (mSv \pm SD) & Prot. B (mSv \pm SD) & Prot. C (mSv \pm SD) & Prot. D (mSv \pm SD) \\
\hline & & Standard & $1.09 \pm 0.35$ & $0.42 \pm 0.21$ & $0.75 \pm 0.35$ & $0.24 \pm 0.35$ \\
& Second trimester & $-10 \%$ & $0.57 \pm 0.18$ & $0.22 \pm 0.10$ & $0.40 \pm 0.19$ & $0.12 \pm 0.16$ \\
Fetal dose & & Optimal & $0.19 \pm 0.08$ & $0.08 \pm 0.02$ & $0.18 \pm 0.06$ & $0.05 \pm 0.06$ \\
& & Standard & $1.49 \pm 0.48$ & $0.59 \pm 0.25$ & $1.05 \pm 0.45$ & $0.34 \pm 0.46$ \\
& \multirow{3}{*}{ Third trimester } & $-10 \%$ & $0.80 \pm 0.24$ & $0.33 \pm 0.13$ & $0.57 \pm 0.25$ & $0.17 \pm 0.22$ \\
& & Optimal & $0.27 \pm 0.12$ & $0.12 \pm 0.05$ & $0.24 \pm 0.11$ & $0.06 \pm 0.07$ \\
\hline
\end{tabular}

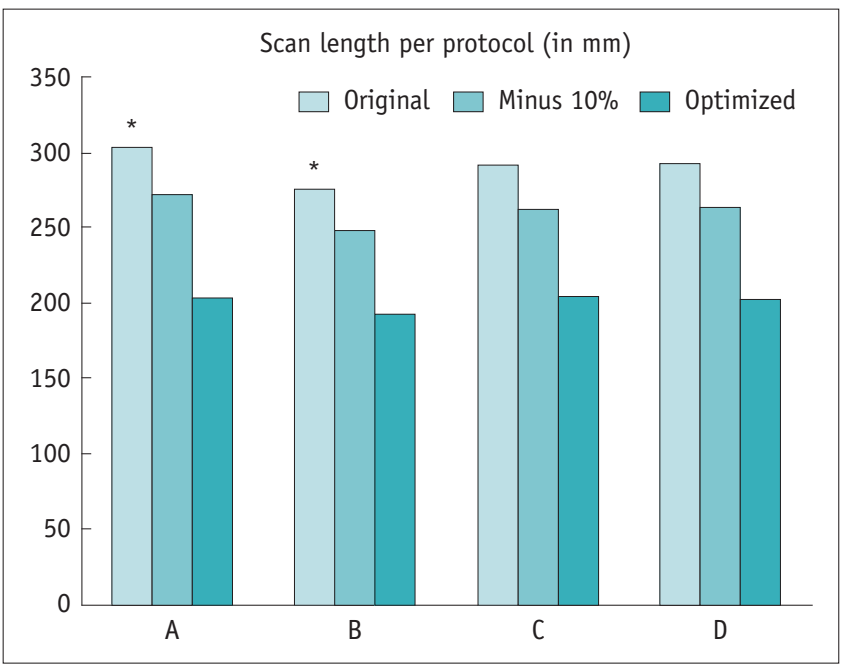

Fig. 4. Mean original scan length ( $\mathrm{mm})$, after $10 \%$ caudal range reduction and per-patient optimization, based on 120 original datasets. *There was significant difference between protocol $\mathrm{A}$ and $\mathrm{B}(p=0.004)$ in original scan length, which was carried through in $10 \%$ range reduction. There was no significant difference between different protocols after individual optimization $(p=0.438)$.

pregnant female phantoms, the effective dose reduction was $26 \%, 25 \%, 26 \%$, and $23 \%$ for protocols $A, B, C$, and $D$, respectively. The overall maternal dose for each of the pregnancy trimesters was reduced by $26-27 \%$ for protocols $A, C$, and $D$, and by $25-26 \%$ for protocol B.
For most of the female adult organs, the individualized scan range resulted in modest mean dose savings: the greatest reduction was $5 \%$ for the thymus (protocol C), $8 \%$ for the lungs (protocol C), and $2 \%$ for breast tissue (protocol A). However, the thyroid gland exhibited more significant dose reductions after z-axis optimization: $45 \%$ for protocol A, $25 \%$ for protocol B, $31 \%$ for protocol $C$, and $60 \%$ for protocol D (Table 3 ).

The larger caudal reduction of the scan range was paramount in reducing the fetal radiation dose (percentages were similar for the 2nd and 3rd trimesters); $82-83 \%$ in protocol $A, 80-81 \%$ in protocol $B, 76-77 \%$ in protocol C, and $79-82 \%$ in protocol $D$ (Table 4 ).

\section{Original Datasets Diagnosis and Image Quality}

On the original 120 CTPA scans, 19 cases of PE were found (16\%); 7 central emboli, 3 lobar emboli, 4 segmental emboli, and 5 cases of exclusively subsegmental PE. None of the PE diagnoses were missed on the CTPA with an optimized scan range; even the exclusively subsegmentally located PE remained visible in these cases. Optimization of the z-axis resulted in four missed incidental findings in the thorax; two cases of missed benign thoracic findings (pulmonary infarction and a small amount of pleural effusion), and two cases of nonspecific pulmonary nodules. 


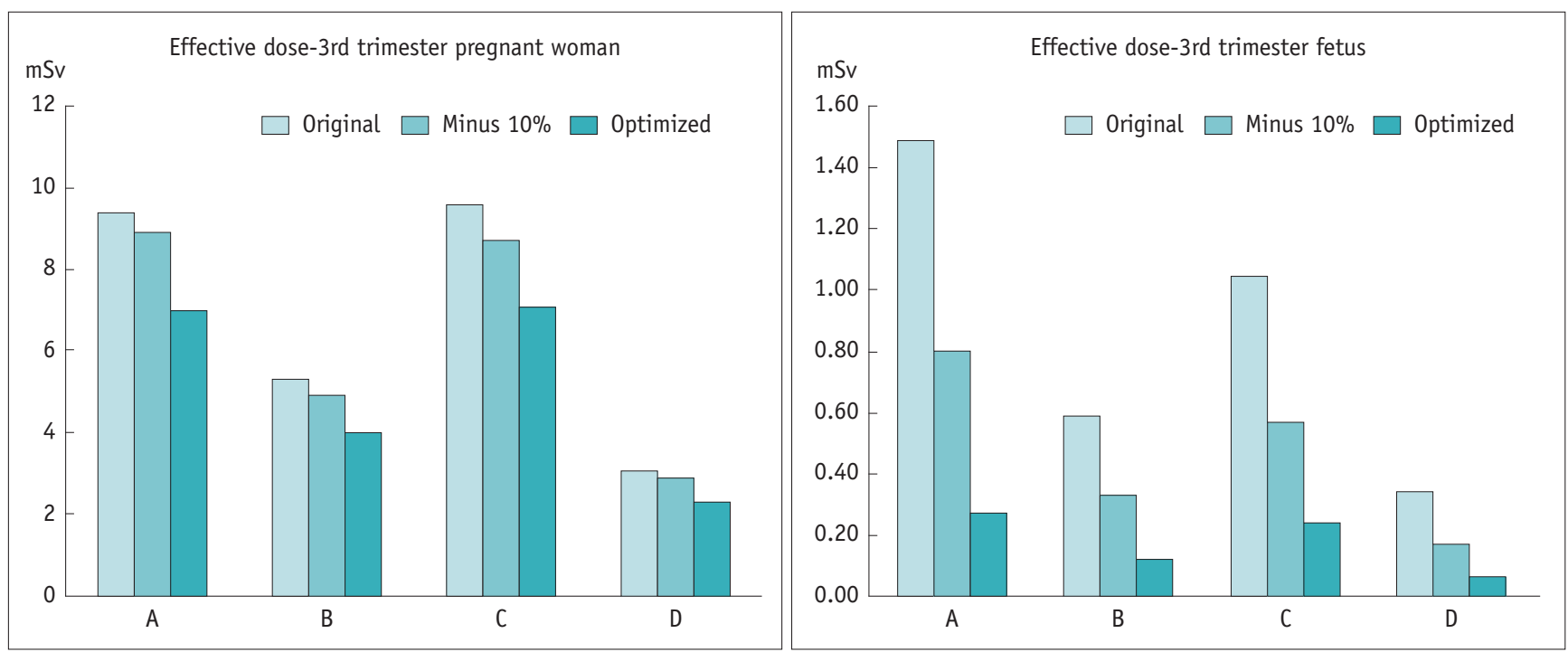

Fig. 5. Scan length adaptations for 3rd trimester pregnant women and fetuses (mean effective dose [mSv] per scan protocol). Graphs illustrate impact of optimizing scan length for different scanners and protocols.

The mean pulmonary enhancement was $335 \pm 92 \mathrm{HU}$ for protocol A, $380 \pm 107 \mathrm{HU}$ for protocol B, $435 \pm 126 \mathrm{HU}$ for protocol C, and $347 \pm 73 \mathrm{HU}$ for protocol $D$. There was a statistically significant difference in attenuation between protocols $A$ and $C(p=0.001)$ and between protocols $C$ and $D(p=0.006)$. Two scans were considered non-diagnostic, with mean pulmonary arterial enhancement of < $180 \mathrm{HU}$, which was likely due to low cardiac output and markedly high body weight; one for protocol A and one for protocol B.

\section{Assessment of Clinical Practice}

Initially, the average scan range selected by the technicians was $236 \pm 35 \mathrm{~mm}$. After observing the results of the study, the technicians set narrower scan ranges in terms of the z-axis, with an average decrease of $65 \pm 40 \mathrm{~mm}$. Interestingly, the $z$ - axis decrease ranged from -17.0-114 $\mathrm{mm}$, indicating that one technician opted to increase the z-axis after the study.

\section{DISCUSSION}

This study clearly demonstrates large variations in the CTPA radiation dose between several CT scanners and scan protocols. In this study, a clear advantage of low $\mathrm{kV}$ scanning in terms of patient dose was observed; the addition of iterative reconstruction in protocol $D$ ensured diagnostic image quality throughout all scans. The optimized scan range allowed for a mean scan range reduction of $30-33 \%$, translating into a $23-27 \%$ effective dose reduction for pregnant women. The dose savings were comparable for each of the tested protocols, indicating that this is a highly effective technique for reducing the CTPA radiation dose with any scanner or protocol. The large dose reduction estimated for the fetuses (up to $83 \%$ ) is explained by the increased distance between fetus and the scanner beam, which effectively reduces the overall radiation absorption $(25,26)$.

One issue, inherent to helical MDCT scanning, is overranging (27). This occurs when the scan length is automatically extended beyond the planned image boundaries, in order to reconstruct the first and last slices of a helical CT scan (28). The Radimetrics ${ }^{\mathrm{TM}}$ software uses the dose-length-product and CT-dose-index (CTDI) or CTDI as reported by the scanner, thereby calculating the actual irradiated length and including any dose from overranging in the effective dose estimations. The two DSCTs used in this study were equipped with fast moving collimators that minimize overranging and the associated radiation dose; however, it is known that for MDCTs without sliding collimators, overranging can constitute a significant portion of the total radiation dose. For the 64-slice MDCT system used in this study, overranging was responsible for adding approximately $1 \mathrm{mSv}$ to the total effective dose.

Currently, hospitals function according to the 'as low as reasonably achievable' or ALARA principle, aiming to make the best choices in scan protocol, CM application, image quality, and more. This principle becomes of even greater importance when performing $\mathrm{CT}$ on younger patients, due to 
the greater hypothetical lifetime attributable risk $(29,30)$.

In this study, no PE diagnoses would have been missed with an optimized scan length. Even if one would miss a solitary subsegmental clot, the clinical relevance of such $\mathrm{PE}$ has yet to be demonstrated and the positive predictive value of CTPA during pregnancy beyond the segmental level remains unclear (31-34). As a general rule, but even more important when imaging pregnant or young patients, radiation dose risks and the need for diagnostic imaging should be weighed in an individual risk assessment (35).

Several studies have been published on decreased scan length in CTPA, proposing rigorous scan length reductions $(14-16 \mathrm{~cm})(36-38)$. These studies demonstrated a statistically and clinically insignificant number of missed PEs (0-0.8\%), which could be taken as an indication that this method is safe for the patients. The current study confirmed this safety in a markedly larger population with a higher prevalence of PE (16\%). Additionally, this is the first study to estimate the impact of optimizing the scan length for the fetal dose of CTPA, which remains a topic of concern and debate in current guidelines.

Although several methods for adapting scan length for scanning pregnant patients have been advocated by the previously mentioned study groups, they have not yet been firmly established in the diagnostic routine or been standardized in any guidelines. However, the current study underlines the importance of careful planning and optimizing the scan length for pregnant patients. This proposed technique can be used in combination with other previously described radiation dose reduction methods, such as automated tube current modulation, bismuth breast shields, and lower kV settings $(35,39)$.

Our study has several limitations. First, although this study used real CTPA datasets, the pregnancy stages and fetuses were simulated using phantom models and Monte Carlo code. The reported effective doses and dose savings need to be verified in a real pregnant patient population. Second, this study included several different CM injection protocols. However, the heterogeneity of injection protocols might not impact on the main outcomes of this study, which focused on the radiation dose rather than the CM dose. Additionally, the simulation software did not account for the increased radiation dose uptake in tissues with high iodine uptake, as described by Perisinakis et al. (40). Lastly, the dual-energy scan protocol had not been optimized in terms of radiation dose.

In conclusion, radiologists and technologists alike should be aware of the variations in radiation dose between different scanners and CTPA scan protocols and their impacts on pregnant women. Optimized scan length settings for each patient can result in significant radiation dose savings for pregnant women and their fetuses.

\section{Conflicts of Interest}

The authors have no financial conflicts of interest.

ORCID

Babs M.F. Hendriks

https://orcid.org/0000-0001-9163-337X

\section{REFERENCES}

1. Kobayashi T, Nakabayashi M, Ishikawa M, Adachi T, Kobashi $G$, Maeda $M$, et al. Pulmonary thromboembolism in obstetrics and gynecology increased by 6.5 -fold over the past decade in Japan. Circ J 2008;72:753-756

2. McLintock C, Brighton T, Chunilal S, Dekker G, McDonnell $\mathrm{N}$, McRae S, et al.; Councils of the Society of Obstetric Medicine of Australia and New Zealand; Australasian Society of Thrombosis and Haemostasis. Recommendations for the diagnosis and treatment of deep venous thrombosis and pulmonary embolism in pregnancy and the postpartum period. Aust N Z J Obstet Gynaecol 2012;52:14-22

3. Shakespeare J, Knight M. Maternal health in pregnancy: messages from the 2014 UK Confidential Enquiry into Maternal Death. Br J Gen Pract 2015;65:444-445

4. Heit JA, Kobbervig CE, James AH, Petterson TM, Bailey KR, Melton LJ 3rd. Trends in the incidence of venous thromboembolism during pregnancy or postpartum: a 30-year population-based study. Ann Intern Med 2005;143:697-706

5. Izadi M, Alemzadeh-Ansari MJ, Kazemisaleh D, MoshkaniFarahani M, Shafiee A. Do pregnant women have a higher risk for venous thromboembolism following air travel? Adv Biomed Res 2015;4:60

6. Leung AN, Bull TM, Jaeschke R, Lockwood CJ, Boiselle PM, Hurwitz LM, et al. American Thoracic Society documents: an official American Thoracic Society/Society of Thoracic Radiology clinical practice guideline--Evaluation of suspected pulmonary embolism in pregnancy. Radiology 2012;262:635646

7. Linnemann B, Bauersachs R, Rott H, Halimeh S, Zotz R, Gerhardt $A$, et al. Diagnosis of pregnancy-associated venous thromboembolism - position paper of the Working Group in Women's Health of the Society of Thrombosis and Haemostasis (GTH). Vasa 2016;45:87-101

8. Jordan EJ, Godelman A, Levsky JM, Zalta B, Haramati LB. CT pulmonary angiography in pregnant and postpartum women: low yield, high dose. Clin Imaging 2015;39:251-253

9. Winer-Muram HT, Boone JM, Brown HL, Jennings SG, Mabie 
WC, Lombardo GT. Pulmonary embolism in pregnant patients: fetal radiation dose with helical CT. Radiology 2002;224:487492

10. Gilet AG, Dunkin JM, Fernandez TJ, Button TM, Budorick $\mathrm{NE}$. Fetal radiation dose during gestation estimated on an anthropomorphic phantom for three generations of CT scanners. AJR Am J Roentgenol 2011;196:1133-1137

11. Hendriks BM, Kok M, Mihl C, Bekkers SC, Wildberger JE, Das M. Individually tailored contrast enhancement in CT pulmonary angiography. Br J Radiol 2016;89:20150850

12. Shahir K, Goodman LR, Lam CA, Midia EC. Dose reduction of $69 \%$ for computed tomography pulmonary angiography: reduced $z$-axis computed tomography pulmonary angiography retains accuracy in those younger than 40 years. $J$ Comput Assist Tomogr 2013;37:765-769

13. Pontana F, Pagniez J, Duhamel A, Flohr T, Faivre JB, Murphy $C$, et al. Reduced-dose low-voltage chest CT angiography with Sinogram-affirmed iterative reconstruction versus standarddose filtered back projection. Radiology 2013;267:609-618

14. Lu GM, Luo S, Meinel FG, McQuiston AD, Zhou CS, Kong X, et al. High-pitch computed tomography pulmonary angiography with iterative reconstruction at $80 \mathrm{kVp}$ and $20 \mathrm{~mL}$ contrast agent volume. Eur Radiol 2014;24:3260-3268

15. Gill MK, Vijayananthan A, Kumar G, Jayarani K, Ng KH, Sun Z. Use of $100 \mathrm{kV}$ versus $120 \mathrm{kV}$ in computed tomography pulmonary angiography in the detection of pulmonary embolism: effect on radiation dose and image quality. Quant Imaging Med Surg 2015;5:524-533

16. Li X, Ni QQ, Schoepf UJ, Wichmann JL, Felmly LM, Qi L, et al. 70-kVp high-pitch computed tomography pulmonary angiography with $40 \mathrm{~mL}$ contrast agent: initial experience. Acad Radiol 2015;22:1562-1570

17. Wichmann JL, Hu X, Kerl JM, Schulz B, Frellesen C, Bodelle B, et al. $70 \mathrm{kVp}$ computed tomography pulmonary angiography: potential for reduction of iodine load and radiation dose. $J$ Thorac Imaging 2015;30:69-76

18. Brenner D, Elliston C, Hall E, Berdon W. Estimated risks of radiation-induced fatal cancer from pediatric CT. AJR Am J Roentgenol 2001;176:289-296

19. Remy-Jardin M, Remy J. Spiral CT angiography of the pulmonary circulation. Radiology 1999;212:615-636

20. Stabin MG, Watson EE, Cristy M, Ryman JC, Eckerman KF, Davis JL, et al. Mathematical models and specific absorbed fractions of photon energy in the nonpregnant adult female and at the end of each trimester of pregnancy. Oak Ridge, TN: Oak Ridge National Lab., 1995

21. Xu XG. An exponential growth of computational phantom research in radiation protection, imaging, and radiotherapy: a review of the fifty-year history. Phys Med Biol 2014;59:R233-R302

22. Kuzo RS, Pooley RA, Crook JE, Heckman MG, Gerber TC. Measurement of caval blood flow with MRI during respiratory maneuvers: implications for vascular contrast opacification on pulmonary CT angiographic studies. AJR Am J Roentgenol
2007;188:839-842

23. ICRP publication 103. The 2007 Recommendations of the International Commission on Radiological Protection. Ann ICRP 2007;37:1-332

24. Ozawa $Y$, Hara M, Shibamoto $Y$. The frequency of insufficient contrast enhancement of the pulmonary artery in routine contrast-enhanced chest CT and its improvement with an increased injection rate: a prospective study. $J$ Thorac Imaging 2011;26:42-47

25. Kelaranta A, Kaasalainen T, Seuri R, Toroi P, Kortesniemi M. Fetal radiation dose in computed tomography. Radiat Prot Dosimetry 2015;165:226-230

26. Goldberg-Stein SA, Liu B, Hahn PF, Lee SI. Radiation dose management: part 2, estimating fetal radiation risk from $\mathrm{CT}$ during pregnancy. AJR Am J Roentgenol 2012;198:W352-W356

27. Kalra MK, Maher MM, Toth TL, Hamberg LM, Blake MA, Shepard $\mathrm{JA}$, et al. Strategies for $\mathrm{CT}$ radiation dose optimization. Radiology 2004;230:619-628

28. Schilham A, van der Molen AJ, Prokop M, de Jong HW. Overranging at multisection CT: an underestimated source of excess radiation exposure. Radiographics 2010;30:1057-1067

29. National Research Council. Health risks from exposure to low levels of ionizing radiation: BEIR VII Phase 2. Washington, DC: National Academies Press, 2006

30. Pearce MS, Salotti JA, Little MP, McHugh K, Lee C, Kim $\mathrm{KP}$, et al. Radiation exposure from $\mathrm{CT}$ scans in childhood and subsequent risk of leukaemia and brain tumours: a retrospective cohort study. Lancet 2012;380:499-505

31. Carrier M, Righini M, Wells PS, Perrier A, Anderson DR, Rodger $M A$, et al. Subsegmental pulmonary embolism diagnosed by computed tomography: incidence and clinical implications. A systematic review and meta-analysis of the management outcome studies. J Thromb Haemost 2010;8:1716-1722

32. Stein PD, Goodman LR, Hull RD, Dalen JE, Matta F. Diagnosis and management of isolated subsegmental pulmonary embolism: review and assessment of the options. Clin Appl Thromb Hemost 2012;18:20-26

33. Hutchinson BD, Navin P, Marom EM, Truong MT, Bruzzi JF. Overdiagnosis of pulmonary embolism by pulmonary CT angiography. AJR Am J Roentgenol 2015;205:271-277

34. Simcox LE, Ormesher L, Tower C, Greer IA. Pulmonary thrombo-embolism in pregnancy: diagnosis and management. Breathe (Sheff) 2015;11:282-289

35. Patel SJ, Reede DL, Katz DS, Subramaniam R, Amorosa JK. Imaging the pregnant patient for nonobstetric conditions: algorithms and radiation dose considerations. Radiographics 2007; 27:1705-1722

36. Shahir K, McCrea JM, Lozano LA, Goodman LR. Reduced z-axis technique for CT pulmonary angiography in pregnancy-validation for practical use and dose reduction. Emerg Radiol 2015;22:651-656

37. Kallen JA, Coughlin BF, O'Loughlin MT, Stein B. Reduced Z-axis coverage multidetector CT angiography for suspected acute pulmonary embolism could decrease dose and maintain 
diagnostic accuracy. Emerg Radiol 2010;17:31-35

38. Atalay MK, Walle NL, Egglin TK. Prevalence and nature of excluded findings at reduced scan length CT angiography for pulmonary embolism. J Cardiovasc Comput Tomogr 2011;5:325332

39. Hurwitz LM, Yoshizumi TT, Goodman PC, Nelson RC, Toncheva G, Nguyen GB, et al. Radiation dose savings for adult pulmonary embolus 64-MDCT using bismuth breast shields, lower peak kilovoltage, and automatic tube current modulation. AJR Am
J Roentgenol 2009;192:244-253

40. Perisinakis K, Tzedakis A, Spanakis K, Papadakis AE, Hatzidakis $A$, Damilakis J. The effect of iodine uptake on radiation dose absorbed by patient tissues in contrast enhanced CT imaging: implications for CT dosimetry. Eur Radiol 2018;28:151-158

41. Hendriks BMF, Eijsvoogel NG, Kok M, Martens B, Wildberger JE, Das M. Optimizing pulmonary embolism computed tomography in the age of individualized medicine: a prospective clinical study. Invest Radiol 2018;53:306-312 